\title{
THEORETICAL EVALUATION OF NONLINEAR EFFECTS ON OPTICAL WDM NETWORKS WITH VARIOUS FIBER TYPES
}

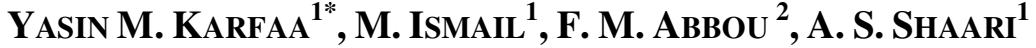 \\ ${ }^{I}$ Department of Electrical, Electronics and Systems Engineering, Universiti Kebangsaan \\ Malaysia, 43600 UKM, Bangi, Selangor, Malaysia. \\ ${ }^{2}$ Faculty of Engineering, Multimedia University, 63100 Cyberjaya, Selangor, Malaysia. \\ *yasin_m_k@yahoo.com
}

\begin{abstract}
A theoretical study is carried out to evaluate the performance of an optical wavelength division multiplexing (WDM) network transmission system in the presence of crosstalk due to optical fiber nonlinearities. The most significant nonlinear effects in the optical fiber which are Cross-Phase Modulation (XPM), Four-Wave Mixing (FWM), and Stimulated Raman Scattering (SRS) are investigated. Four types of optical fiber are included in the analysis; these are: single-mode fiber (SMF), dispersion compensation fiber (DCF), non-zero dispersion fiber (NZDF), and non-zero dispersion shifted fiber (NZDSF). The results represent the standard deviation of nonlinearity induced crosstalk noise power due to FWM and SRS, XPM power penalty for SMF, DCF, NZDF, and NZDSF types of fiber, besides the Bit Error Rate (BER) for the three nonlinear effects using standard fiber type (SMF). It is concluded that three significant fiber nonlinearities are making huge limitations against increasing the launched power which is desired, otherwise, lower values of launched power limit network expansion including length, distance, covered areas, and number of users accessing the WDM network, unless suitable precautions are taken to neutralize the nonlinear effects. Besides, various fiber types are not behaving similarly towards network parameters.
\end{abstract}

KEYWORD: Nonlinear effects, Nonlinearity standard deviation, BER, Power penalty, and WDM.

\section{INTRODUCTION}

High capacity long reach transmission systems employ a large number of wavelength division multiplexed (WDM) channels with dense channel spacing and high bit rate per channel. In conventional WDM systems, the main transmission impairments are fiber losses, group velocity dispersion (GVD) and nonlinear fiber effects [1]. Due to the desired long distance between amplifiers, high channel powers are required in order to achieve sufficiently large signal-to-noise ratios (OSNR). The use of erbium doped fiber amplifiers (EDFA) allows overcoming the first limitation while the others are usually minimized by optimizing the dispersion compensation with a proper management. All these system requirements give rise to nonlinear impairments. In long distance transmission links above $100 \mathrm{~km}$ including analog signals over optical fibers, high transmission powers are involved which give rise to nonlinear effects such as self-phase modulation (SPM) in a single channel system, cross phase modulation (XPM), four wave mixing (FWM), 
stimulated Raman scattering (SRS), and stimulated Brillouin scattering (SBS) between channels in a WDM system and can be highly detrimental in the presence of dispersion [2]. These nonlinear effects can limit performance in both digital and analog systems.

\section{SYSTEM THEORETICAL ANALYSIS}

Avoid using tab or enter buttons whenever possible. (Normal) (what is this??). Figure 1 below shows a typical $W_{C}$ channels optical WDM system. It is assumed that the network is experiencing all the three nonlinear effects under study; these are XPM, FWM, and SRS. For this network, the analysis of all the nonlinear effects is possible by taking their effects on pulses propagating inside an optical fiber and to be included by using the nonlinear Schrödinger Equation (NLSE), which is used to describe the slowly varying complex envelope of the optical field when expressing any of the nonlinear effects in the form that is given by [3] as in Eq. (1) below:

$$
\frac{\partial A_{1}}{\partial z}+\frac{\alpha_{1}}{2} A_{1}+\frac{i}{2} \beta_{21} \frac{\partial^{2} A_{1}}{\partial T^{2}}-\frac{1}{6} \beta_{31} \frac{\partial^{3} A_{1}}{\partial T^{3}}=i \gamma_{1}\left[9\left|A_{1}\right|^{2}\left|A_{2}\right|^{2}\left|A_{3}\right|^{2}\right] A_{1}+\psi_{\omega}
$$

Since three of the nonlinear effects are considered here, so, Eq. (1) contains a last term on the R.H.S $\left(\psi_{\omega}\right)$ to account for SPM and SBS that are not included in this study; where $\alpha_{n}$ represents the $\mathrm{n}^{\text {th }}$ channel's attenuation parameter, $\beta_{2 n}$ represents the $\mathrm{n}^{\text {th }}$ channel's second order dispersion parameter, $\beta_{3 n}$ represents the $\mathrm{n}^{\text {th }}$ channel's third order dispersion parameter, $\gamma_{n}$ represents the $\mathrm{n}^{\text {th }}$ channel's nonlinearity coefficient where $\left.\gamma=n_{2} \omega_{0} / c A_{\text {eff }}\right), T_{R n}$ represents the $\mathrm{n}^{\text {th }}$ channel's Raman shift, ${ }_{m n}$ represents the walk-off parameter between channels $m$ and $n$.

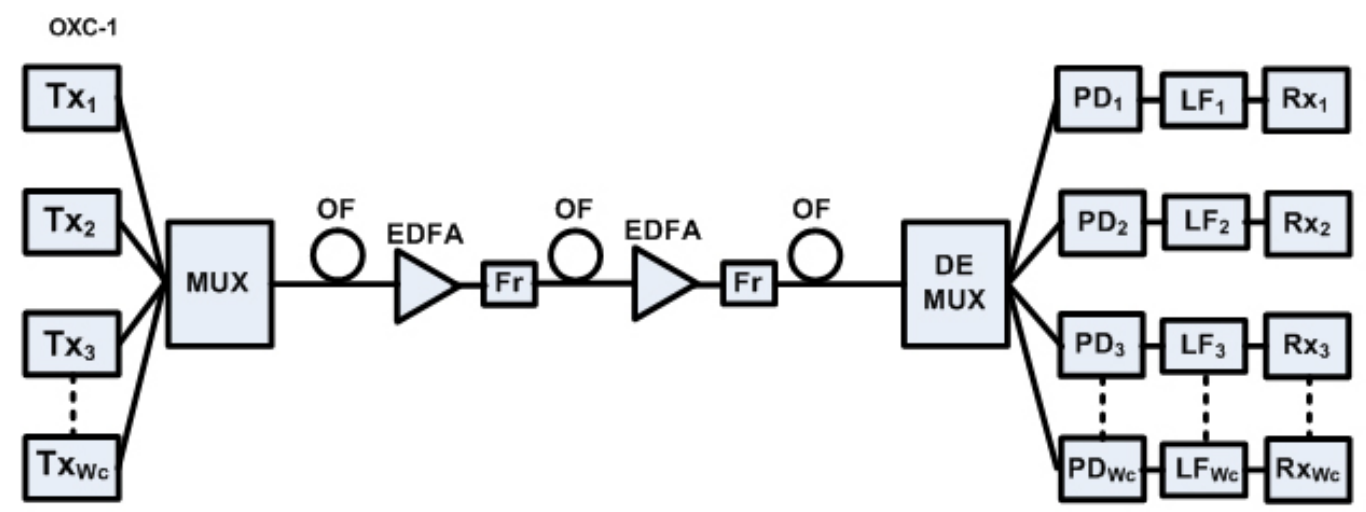

Fig. 1: Optical Communication System for Multiple Transmitted Channels and Various Types of Fiber (OF: optical fiber, can be SMF, DCF, NZDF, or NZDSF).

\subsection{Theoretical Analysis of XPM}

XPM is a fatal nonlinear impairment in WDM systems as a nonlinear phase noise results after the interaction of optical amplified spontaneous emission (ASE) and fiber Kerr nonlinearities [4]; it is one of the dominant degradation effects for bit rates as much as $10 \mathrm{Gbps}$ in WDM systems although it can be of use as in case of the optical signal regeneration for future high-speed optical communication systems; when the interaction 
takes place between continuous optical waves, constant phase changes and constant polarization rotations are induced, but, if the intense wave is pulsed or intensity modulated, the induced nonlinear phase shift becomes time dependent, leading to phase modulation and polarization modulation of the co-propagating waves. Thereby, new spectral components are generated on their optical spectra resulting in an XPM-induced spectral broadening. In some researches where the channel spacing is reduced, the degradation due to XPM and FWM increased and the optimum dispersion compensation scheme is significantly affected by these effects. The dispersion is the middle parameter that increasing it minimizes FWM but increases XPM and vice versa. Therefore, since FWM can be minimized using a high local dispersive transmission fiber such as a SMF and thus the main inter-channel nonlinear effect is XPM. The mathematical expression to calculate XPM induced crosstalk power is derived following literature models [5-7]. The $\mathrm{XPM}$ power fluctuation due to one channel interference is [5]:

$$
P_{j, X, k}(\omega, L)=4 \gamma P_{j 0} P_{k}(\omega) e^{-\alpha L} \frac{\sin \left(D_{C} \omega^{2} / 2\right)}{\alpha+j \beta_{2}(k \Delta \omega) \omega}
$$

where $\Delta \lambda=\Delta \omega \lambda^{2} / c$ is the wavelength spacing, and $D_{C}=-2 \pi c \beta_{2} / \lambda^{2}$ is the chromatic dispersion, $\beta_{2}$ is the group velocity dispersion. By taking the magnitude and ignoring $\alpha^{2}$ being too small:

$$
\left|P_{j, X, k}(\omega, L)\right|=8 \pi \gamma P_{j 0} P_{k}(\omega) e^{-\alpha L} \frac{\sin \left(D_{C} \omega^{2} / 2\right)}{D_{C} k \Delta \lambda \omega}
$$

Considering the contributions from $W_{C}$ number of channels, Eq. (2) can be rewritten as:

$$
\left|P_{j, X, k}(\omega, L)\right|=\sum \frac{1}{W_{C}} 8 \pi \gamma P_{j 0} P_{k}(\omega) e^{-\alpha L} \frac{\sin \left(D_{C} \omega^{2} / 2\right)}{D_{C} k \Delta \lambda \omega}
$$

The total noise variance due to XPM-induced power fluctuation for an IM/DD system $P_{X P M}$ is obtained by applying the integration of Eq. (4) for the interval $\left(\omega_{1}, \omega_{2}\right)$ as follows:

$$
P_{X P M}=R_{d} \int_{\omega_{1}}^{\omega_{2}}\left|\Delta P_{j, X, k}(\omega, L)\right|^{2}|H(\omega)|^{2} d \omega
$$

\subsection{Theoretical Analysis of FWM}

FWM occurs when the channels in a WDM system are equally spaced, so that the new waves generated by FWM will fall at channel frequencies and, thus, will give rise to crosstalk; its severity is maximized when dispersion is minimized by some proper techniques, so they are inversely proportional because the FWM products add coherently in each span. FWM may result when three light signals at different wavelengths interact in the fiber to create a fourth light signal at either a different or same wavelength causing a distortion to the desired signal. The new optical waves frequencies are judged by $f_{i j k}=f_{i}+f_{j^{-}}$ $f_{k}$, which is $\theta^{+}$; or it results when two propagating waves at frequencies $f_{1}$ and $f_{2}$ mix and generate sidebands at $2 f_{1}-f_{2}$ and $2 f_{2}-f_{1}$, which is $\theta$. These sidebands co-propagate with initial waves and grow at their expense, besides creating inband crosstalk that cannot be 
filtered optically or electronically and channels beat against each other to form intermodulation products. The mathematical expression to calculate FWM induced crosstalk power is derived with following the models done by [8-11]:

$$
P_{F W M}=\sum_{i j k}\left[\frac{4 \times 10^{4} f_{i j k} \lambda G_{i j k}^{2} L^{2}\left(N_{A}\right)^{1 / 2} U_{i j k}}{9 c A_{e f f} \Delta \omega^{2}\left|D_{C}\right|\left(\alpha^{2}+\Delta \beta_{i j k}^{2}\right)}\right]^{1 / 3} \gamma P^{2}
$$

where $f_{i j k}$ is the frequency for channels $i, j, k$ respectively, $\lambda$ is the operating wavelength of the optical signal, $G_{j k l}=1$ for two-tone products, $G_{j k l}=2$ for three-tone products, $L$ is the length of fiber line, $N_{A}$ is the number of EDFAs, $U_{i j k}$ is the degeneracy factor and takes values of 3 or $6, c$ is the speed of light in free space, $A_{\text {eff }}$ is the fiber core effective cross-section area, $\Delta f$ is the channel spacing, $D_{C}$ is the chromatic dispersion, $\Delta \beta$ is the phase matching coefficient for FWM to occur. Figure 2 below shows the power spectral representation of the FWM, XPM, and SRS nonlinear effects as it appears in the output for a regular input to the system when $W_{C}$ frequency channels are transmitted in optical WDM system.

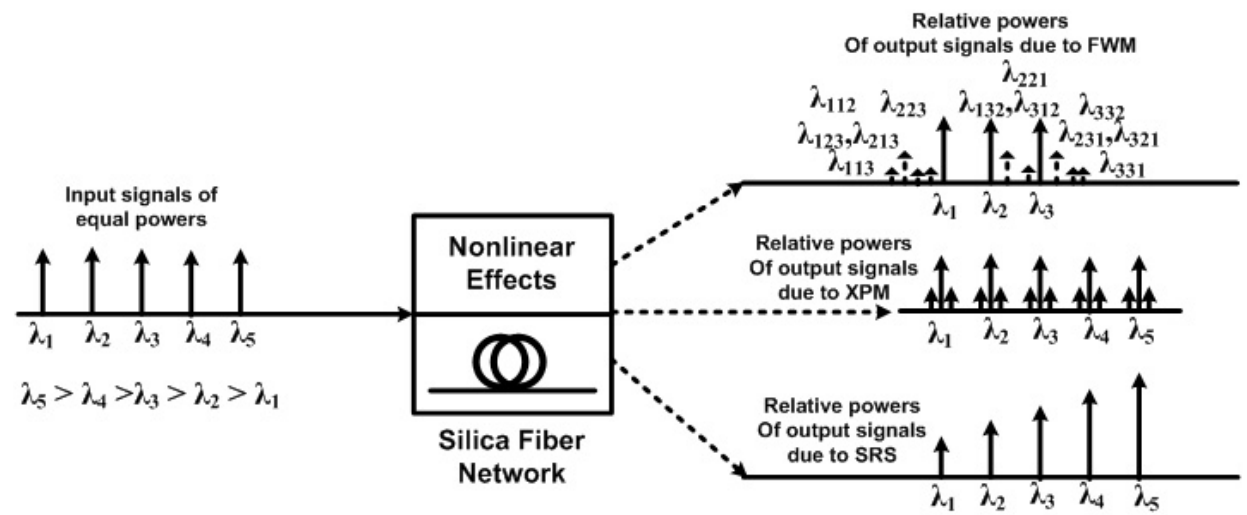

Fig. 2: Nonlinear Impairments in an Optical WDM Network as a Limiting Power Degradation Factor.

\subsection{Theoretical Analysis of SRS}

SRS causes the optical power from one mode (with a higher frequency) to be transferred in the forward direction to the same or other modes, at a lower frequency. It depends critically upon the optical power density within the fiber and hence only becomes significant above threshold power levels; therefore, the launched power into the system is limited because of these Raman effects [12]. Beside that, the large-capacity and long haul network transmission essentially use the erbium-doped fiber amplifiers (EDFAs) to compensate the fiber attenuation without using electrical regenerators. The expansion of the optical network dimensions to thousands of kilometers with the feasibility of the concept of transparent optical networks introduces some problems like accumulated ASE noise and aggravation of fiber nonlinearity effects, where SRS is one of them. Even the mitigation techniques of SRS will give some undesired results, for example, the mitigation by having a large core and short fiber which reduces both the optical power density in the 
core and the interaction length of the optical field in the fiber, but this leads to a multimode core, and can consequently lead to a significant degradation of the output beam quality. Other techniques have their drawbacks as well. Anyhow, there are many attractive features as a frequency converter, beam clean-up, pulse compression, etc. For the above network where the occurrence of SRS problem is assumed, the mathematical expression to calculate SRS induced crosstalk power is derived with following the models done by [13$16]$.

When channel $O$ with the shortest wavelength $\lambda_{\text {shortest }}$ is affected by a longer $\lambda_{\text {longest }}$ denoted by channel $i$, the probabilities for the channels in ON state are:

$$
\begin{aligned}
& P_{0} O(\text { channel } O \text { is } \mathrm{ON})=P_{O N} \\
& P_{i} O(\text { channel } i \text { is } \mathrm{ON})=P_{O N}
\end{aligned}
$$

Assuming both channels are random and independent from each other, the probability that both channels are in ON state is:

$$
P_{r b O N}=P_{0} O \times P_{i} O=P_{O N} \times P_{O N}=P_{O N}^{2}
$$

SRS happens when both channels involved are in ON state, of which the probability is shown as above. The average SRS power depletion in channel 0 due to interference from channel $i, \mu_{D 0 i}$ is defined as:

$$
\mu_{D 0 i}=D_{P 0} \times P_{r b O N}
$$

where $D_{P 0}$ is the fractional power lost by channel $O$ due to all other channels, and is defined as:

$$
D_{P 0}=\sum_{i=1}^{W_{C}-1} 1.4 \times 10^{-6} \times \frac{W_{C} P_{i} O L_{\text {eff }}}{A_{\text {eff }}} \sqrt{\frac{\Delta \omega \lambda_{i} \gamma_{i}}{4 \pi \lambda_{0}}}
$$

where $W_{C}$ is the wavelength channels number, $P_{i}$ is the optical power carried in the $\mathrm{i}^{\text {th }}$ channel, $P_{i} O$ is the $P_{i}$ at $\mathrm{ON}$ state, $A_{\text {eff }}$ is the effective core area of the fiber, $L_{\text {eff }}$ is the effective fiber length given by $L_{\text {eff }}=(1-\exp (-\alpha L)) / \alpha$, where $\alpha$ is the fiber loss coefficient and $L$ is the fiber length. The Raman gain coefficient $\gamma_{i}$ that is coupling the first and $\mathrm{i}^{\text {th }}$ channel can be expressed using the triangular Raman gain as follows:

$$
\gamma_{i}=\frac{i \Delta v}{1.5 \times 10^{13}} \gamma_{P} \text { for } i \Delta v<1.5 \times 10^{13} \text { and } \gamma_{i}=0 \text { otherwise }
$$

Here, $\gamma_{P}$ is the peak Raman gain coefficient with a value of $6 \times 10^{-12} \mathrm{~cm} / \mathrm{W}^{2}$ and $\Delta v$ is the channel frequency spacing. The mean and standard deviation of $D_{P 0}$ are related according to the following relation [16]:

Case I: Walk-off length $L_{W}$ is very large.

$$
\sigma_{D 0 i}=\mu_{D 0 i} \sqrt{\frac{2\left(2 W_{C}-1\right)}{3 W_{C}\left(W_{C}-1\right)}}
$$


Case II: Walk-off length $L_{W}$ is very small.

$$
\sigma_{D 0 i}=\mu_{D 0 i} \sqrt{\frac{\alpha L_{W}}{W_{C}\left(W_{C}-1\right)}}
$$

The total SRS induced crosstalk noise power due to interactions of all channels $P_{S R S}$ is given by:

$$
P_{S R S}=\sum_{i=1}^{W_{C}-1} 2.2 \times 10^{-3} \times \frac{P_{i} O}{A_{\text {eff }}} \sqrt{\frac{W_{C} \lambda_{i} \gamma_{i} L_{e f f}}{2 \lambda_{0}}} \times \sqrt[1 / 4]{\frac{\Delta \omega N_{A}}{2 \pi R_{b}\left|D_{C}\right|}} K_{1}
$$

where $K_{1}=\sqrt[1 / 4]{\frac{2\left(2 W_{C}-1\right)}{3 W_{C}\left(W_{C}-1\right)}}, \sqrt[1 / 4]{\frac{\alpha L_{W}}{W_{C}\left(W_{C}-1\right)}}$ for large and small walk of respectively.

Then, the bit error rate for the system due to nonlinearities is given by [3]:

$$
B E R_{N L}=\frac{1}{4}\left[\operatorname{erfc}\left(\frac{I_{1}-I_{D}}{\left(P_{N L}+\sigma_{A S E_{-} 1}\right) \sqrt{2}}\right)+\operatorname{erfc}\left(\frac{I_{D}-I_{0}}{\sigma_{A S E_{-} 0} \sqrt{2}}\right)\right]
$$

where $P_{N L}$ is to account for the calculated nonlinear crosstalk noise power; so it can be $P_{X P M}, P_{F W M}$, or $P_{S R S} ; I_{D}$ is given by [3]:

$$
I_{D}=\frac{\sigma_{0} I_{1}+\sigma_{1} I_{0}}{\sigma_{0}+\sigma_{1}}
$$

where $I_{1}$ is the transmitted current for bit ' 1 ', $I_{0}$ is the transmitted current for bit ' 0 ', $I_{D}$ is the threshold current level for the decision circuit, $\sigma_{A S E_{-} 1}$ is the Amplified Spontaneous Emission (ASE) noise for transmission of bit ' 1 ', $\quad \sigma_{A S E_{-} 0}$ is the ASE noise for transmission of bit ' 0 '. The accumulated ASE_ASE beat noise for bits ' 1 ' and ' 0 ' is given by [17]:

$$
\sigma_{A S E-A S E}^{2}=4 R_{d}^{2}\left(P_{A S E_{-} a c c n}^{c h}\right)^{2}\left(2 B_{0}-B_{e}\right) \frac{B_{e}}{B_{0}^{2}}
$$

where $R_{d}$ is the photo detector responsivity, $P_{A S E-a c c n}^{c h}$ is the accumulated ASE noise for any channel, $B_{0}$ is the optical bandwidth, and $B_{e}$ is the electrical bandwidth.

\section{MATERIALS AND METHOD}

The first step of the method of evaluating the nonlinear effects is by conducting the theoretical analysis with modeling the optical WDM network under three considered nonlinear effects; these are: XPM, FWM, and SRS. Then, the second step is to use the findings of other researchers for specifying the best model among those. The third step is to derive the relevant expressions for finding the XPM, FWM, and SRS induced crosstalk. The fourth step is to simulate the theoretical expressions of the three nonlinear effects with 
the mathematical calculations using the Matlab software. The fifth step is to analyze the results of the simulations. And the last step is to conclude the outcome of the evaluation of nonlinear effects. For all of the three models of nonlinear crosstalk derivations, the same basic equation is used, which represents the pulses propagating inside, that is the Nonlinear Schrödinger Equation (NSE), which is commonly used to describe the slowly varying complex envelope of the optical field when expressing the XPM, FWM, and SRS in the form [3] (hanging sentence??). With taking NSE equation, then some other models are considered or followed to ease the derivations with some approximations. Those models are indicated to when used. (poorly written; consider rephrasing) The model of this optical WDM system is shown in Fig. 1. This system is suffering from nonlinear effects. Since the figure shows a single-line transmission system with multiple channels, so, it is assumed that at each one certain time, there is a certain type of fiber to be used, and replace by other types alternatively. Tables 1 and 2 for the values of system parameters that are used in the simulation are shown below. (last two sentences are poorly written; consider rephrasing)

Table 1: Input parameters used in the simulation of all nonlinear crosstalk effects.

\begin{tabular}{ll}
\hline Parameter & Values \\
Number of channels, $W_{C}$ & 128 \\
Number of nodes, $N$ & 20 \\
Number of amplifiers, $N_{A}$ & 20 \\
Optical fiber length, $L$ & 200 \\
Input Power, $P_{\text {in }}$ & 0 \\
Bit rate, $R_{b}$ & 10 \\
Optical bandwidth, $B_{o}$ & 20 \\
Electrical bandwidth, $B_{e}$ & 10 \\
Operating frequency, $v$ & $1.9355 \times 10^{14}$ \\
Frequency channel spacing, $\Delta \omega$ & 50 to 100 \\
Wavelength spacing, $\Delta \lambda$ & 0.4 to 0.8 \\
Nonlinear refractive index, $n_{2}$ & $2.6 \times 10^{-20}$ \\
Group index, $n_{g}$ & 1.46 \\
Basic operating light wavelength, $\lambda$ & 1550 \\
Receiver's responsivity, $R_{d}$ & 1 \\
Load resistence, $R_{L}$ & 50 \\
Inversion factor, $F_{e}$ & 3 \\
Amplifier's gain, $G$ & 20 \\
\hline
\end{tabular}


Raman gain coefficient, $g_{R}$

$6 \times 10^{-13}$

Temperature, $T$

300

Table 2: Parametric characteristics of many types of optical fibers.

\begin{tabular}{lllll}
\hline Fiber type & $A_{\text {eff }}\left(\mu \mathrm{m}^{2}\right)$ & $D_{C}(\mathrm{ps} / \mathrm{km} / \mathrm{nm})$ & $\alpha(\mathrm{dB} / \mathrm{km})$ & $\gamma\left(W^{-1} \mathrm{~km}\right)$ \\
SMF & 80 & 17 & 0.2 & 2.5 \\
DCF & 20 & -80 & 0.29 & 3 \\
NZDF & 72 & -3 & 0.23 & 3.9 \\
NZDSF & 50 & 4.5 & 0.25 & 3.84 \\
\hline
\end{tabular}

\section{RESULTS AND DISCUSSION}

A Matlab software is used to validate the three analytical expressions for the nonlinear crosstalk noises XPM, FWM, and SRS. Some of the system parameters used are number of channels, $W_{C}=128$ channels, input power $=0-20 \mathrm{dBm}$, fiber loss coefficient $=0.2-0.25$ $\mathrm{dB} / \mathrm{km}$, channel spacing range $10-100 \mathrm{GHz}(0.08-0.8 \mathrm{~nm})$. The XPM power penalty and crosstalk noises due to FWM, and SRS are shown in Fig. 3, 4 and 5 respectively versus number of channels and input powers respectively. Figure 3 is for the network when it is impaired by XPM, and shows the XPM power penalty versus input power, whereas Fig. 4 shows the standard deviation of FWM induced crosstalk versus number of channels when the optical WDM network is experiencing FWM nonlinear effect. Figure 5 is for the network which is influenced by SRS. Four types of optical fiber are used in the calculations for the graphic relations: single-mode fiber (SMF), dispersion compensation fiber (DCF), non-zero dispersion fiber (NZDF), and non-zero dispersion shifted fiber (NZDSF). These fibers differ from each other in the specifications that are expressed by all the necessary parameters for the computations to satisfy the algorithm of finding the different fiber nonlinearity originated noise phenomenon's (like XPM, FWM, and SRS in this case). The used parameters in the calculations are fiber attenuation, chromatic dispersion, nonlinearity refractive index coefficient, fiber nonlinearity factor, and fiber core cross-sectional area. The differences in the parameters' values among the various fiber types continue to appear finally in the graphic relations of power penalties versus input powers, and so, they vary in the graphs. Using the mathematical meaning for this, it seems that different types of fiber respond differently to the effect of changing the input powers and the relevant changes in the power penalties due to fiber nonlinearities. Lastly, Figure 6 shows BER versus received power in the presence of all the three nonlinear effects: XPM, FWM, and SRS. Standard fiber SMF is used, while the parameters are fixed at 8 nodes, $100 \mathrm{GHz}$ channels spacing, while the rest of the parameters are as in Tables 1 and 2. 


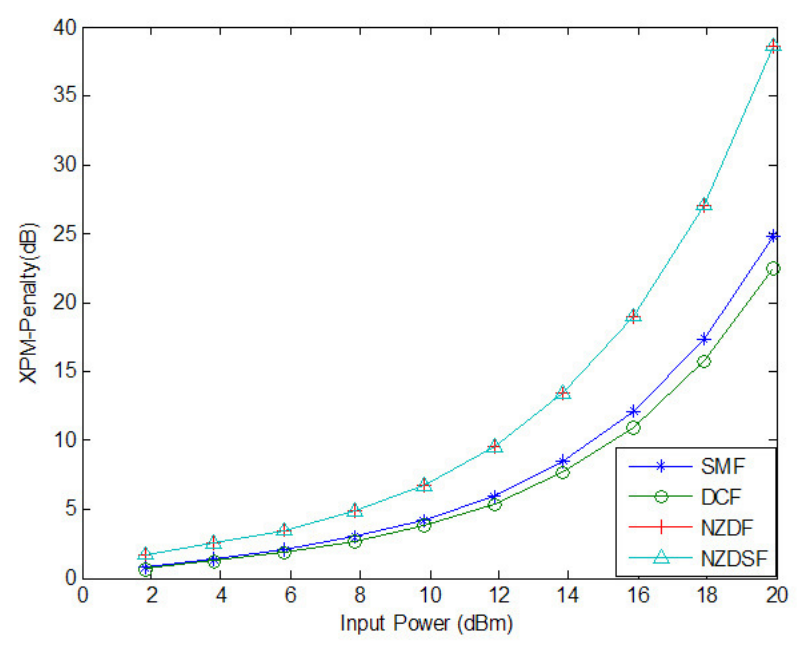

Fig. 3: Penalty due to XPM versus Input Power for various fiber types where ASE noises are not counted.

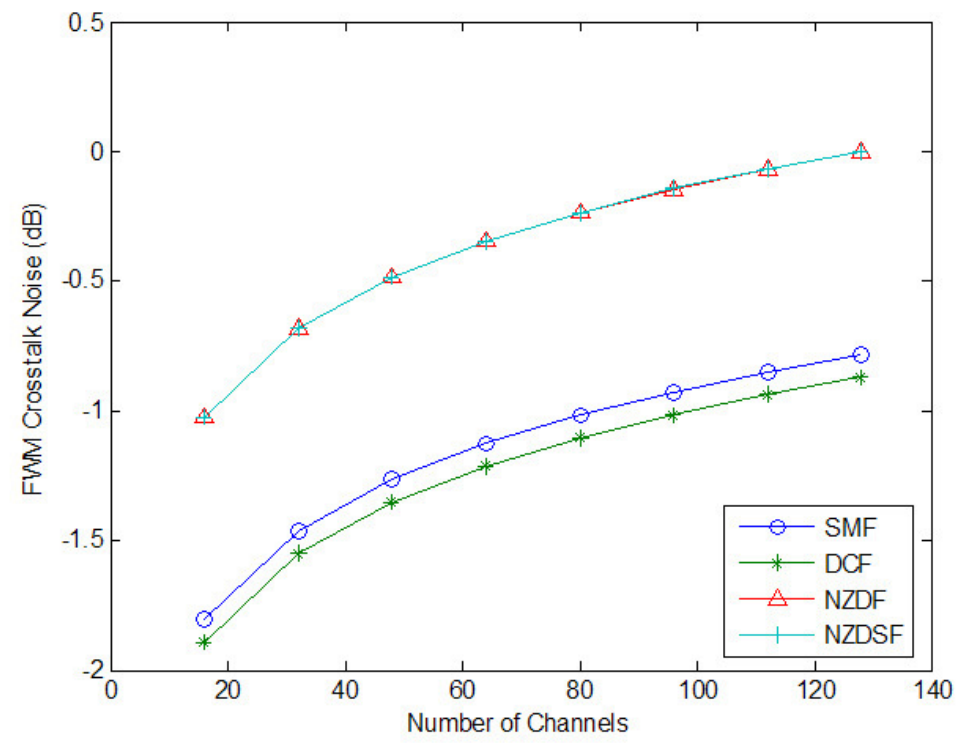

Fig. 4: Standard deviation of FWM induced crosstalk versus Input Power for various fiber types. 


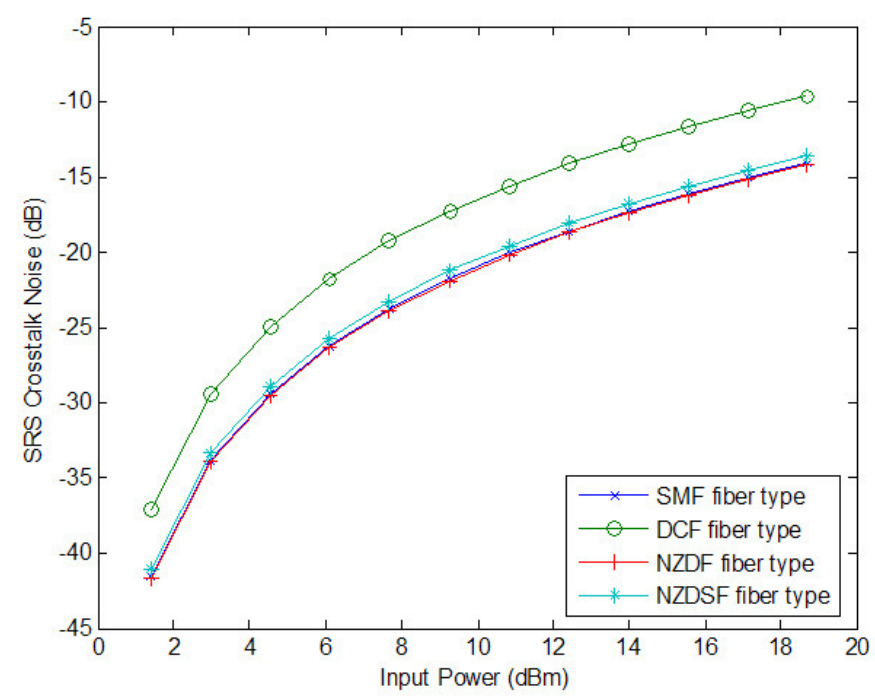

Fig. 5: Standard deviation of SRS induced crosstalk versus Input Power for various fiber types.

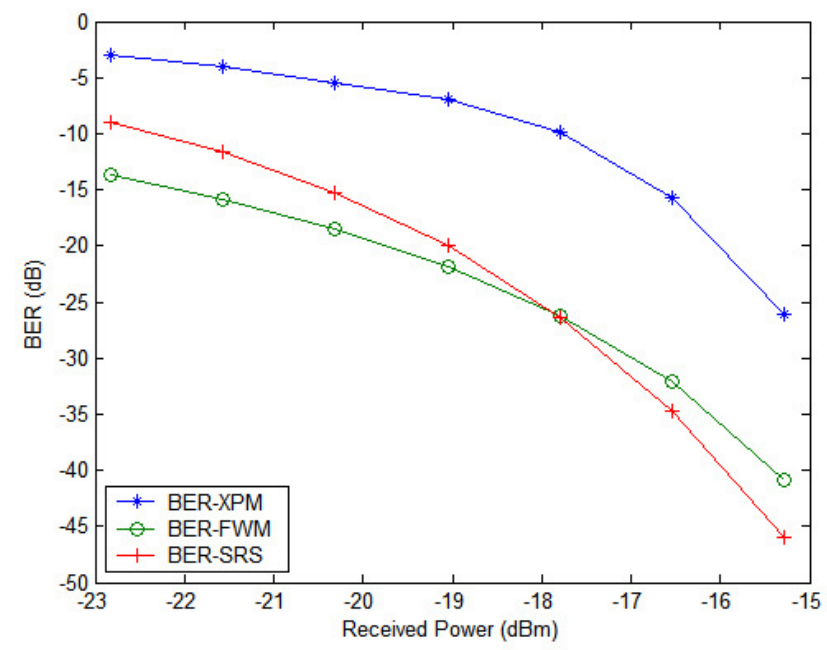

Fig. 6: BER due to nonlinear effects (XPM-FWM-SRS) versus Received Power using standard SMF fiber.

\section{CONCLUSION}

In this paper, a theoretical study is carried out to investigate the transmission of signal for $N$ nodes optical WDM network in the presence of nonlinear crosstalk due to XPM, FWM, and SRS. The performance analysis is done by calculating the XPM power penalty, and the standard deviation of the noises due to FWM and SRS that the optical signal is experiencing when transmitting through 128-144 channels. The power penalty and standard deviation to evaluate the nonlinear crosstalk noise power in the analysis are chosen because those give an indication of signal transmission in a problem of compensation requirement or an extra budget to pay back for the lost power, since the 
BER of the system must be kept at a level of $10^{-9}$ or below, which is preferable as it means that more packets are transmitted with no errors. When the BER is high, the performance of the network deteriorates as it indicates corrupted and lost data. The results using Matlab show that a higher BER and nonlinear crosstalk occurs in a WDM network when the number of nodes is large. This is true for large number of channels; that means small channel spacing (except SRS that reduces with increasing number of channels or reducing channel spacing). The dispersion is working proportionally with XPM and oppositely with FWM and SRS. The noises due to nonlinear effects are not same for different types of fiber, and that indicate the nature of the limitations due to nonlinearity and due to fiber type. Narrowing channel spacing is also not preferable as it increases the BER. In conclusion, to transmit signal in optical WDM networks, physical impairments such as XPM, FWM, and SRS should be taken into account as those directly affect the BER.

The standard single-mode fiber (SMF) is showing better results in the performance evaluation than the other tested three types of fiber (DCF, NZDF, and NZDSF). Then, since the DCF fiber is used in the dispersion management and dispersion map as a solution to go close to the trade-offs in the way to keep the negative effects of the fiber nonlinearities to the minimum at the acceptable level that helps to improve the system's performance, so, this DCF fiber is the best to use in alternative mixing techniques to play this turn.

\section{ACKNOWLEDGEMENT}

Dr. Hairul Azhar Abdul Rashid, the senior lecturer in Faculty of Engineering, Multimedia University kindly provided technical support, printing facilities, downloading reference papers, and many useful discussions.

\section{REFERENCES}

[1] T. Alves, N. Costa, and A.V. Cartaxo, "Dependence of XPM degradation on the dispersionmap for $10 \mathrm{Gbit} / \mathrm{s}$ WDM links over standard-fiber employing duobinary format," In Transparent optical networks, $9^{\text {th }}$ ICTON Intern. Conference. Proc. no.1, 79-82. 2007.

[2] B. S. Marks, C. R. Menyuk, A. L. Campillo, and F. Bucholtz, "Analysis of interchannel crosstalk in a dispersion-managed analog transmission link," Journ. of Lightwave Technol. Vol. 24, no. 6, 2305-2310, 2006.

[3] G. P. Agrawal, "Fiber Optic Communication Systems," John Wiley \& Sons: New York, 2002.

[4] X. Li, F. Zhang, Z. Chen, and A. Xu, "Optimum dispersion mapping of $40 \mathrm{~Gb} / \mathrm{s} \mathrm{RZ-DPSK}$ WDM transmission systems with XPM-induced nonlinear phase noise," In Intern. NanoOptoelectronics Workshop i-NOW '07 Proc. 156-157, 2007.

[5] B. Xu, and M. B. Pearce, "Comparison of FWM- and XPM-induced crosstalk using the Volterra series transfer function method," Journ. of Lightwave Technol. Vol. 21, no. 1, 40-53, 2003.

[6] A. V. T. Cartaxo, "Cross-phase modulation in intensity modulation direct detection WDM systems with multiple optical amplifiers and dispersion compensators," Journ. of Lightwave Technol. 17(2): 178-190, 1999.

[7] I. B. Djordjevic, A. Stavdas, C. Skoufis, S. Sygletos, and C. Matrakidis, "Analytical modeling of fiber non-linearities in amplified dispersion compensated WDM systems," Intern. Journ. of Modeling and Simulation. Vol. 23, no. 4, 226-233, 2003. 
[8] R. Ramaswami, and N. S. Kumar, "Optical networks: a practical perspective," $2^{\text {nd }}$ edition: Academic Press, 2002.

[9] M. Eiselt, "Limits on WDM Systems due to four-wave mixing: a statistical approach," Journ. of Lightwave Technol. Vol. 17, no. 11, 2261-2267, 1999.

[10] S. Song, T. C. Allen, K. R. Demarest, and R. Hui, "Intensity-dependent phase-matching effects on four-wave mixing in optical fibers," Journ. of Lightwave Technol. Vol. 17, no. 11, 2285-2290, 1999.

[11] A. R. Chraplyvy, "Limitations on lightwave communications imposed by optical-fiber nonlinearities," Journ. of Lightwave Technol. Vol. 8, no. 10, 1548-1557, 1990.

[12] M. K. Abdullah, Z. A. T. Al-Qazwini, M. D. A. Samad, and M. Mokhtar, "Optimum transmit power for optical CDMA transmission systems considering stimulated Raman scattering (SRS) effects," In Wireless and Optical Communications Networks WOCN'7 IFIP Intern. Conference. Proc. 1-4, 2007.

[13] A. R. Chraplyvy, "Optical power limits in multi-channel wavelength-division-multiplexed systems due to stimulated Raman scattering," Electronics Lett. Vol. 20, no. 2, 58-59, 1984.

[14] F. Forghieri, R. W. Tkach, and A. R. Chraplyvy," Effect of modulation statistics on Raman crosstalk in WDM systems," IEEE Photon. Technol. Lett. Vol. 1, no. 1, 101-103, 1995.

[15] M. Eiselt, "The impact of non-linear fiber effects on fiber choice for ultimate transmission capacity," In Optical Fiber Communication Conference. Proc. no. 1, 58-60, 2000.

[16] K. P. Ho, "Statistical properties of stimulated Raman crosstalk in WDM systems," Journ. of Lightwave Technol. Vol. 18, no. 7, 915-921, 2000.

[17] G. Keiser, "Optical fiber communications, “ $3^{\text {rd }}$ edition, McGraw-Hill, 2000.

\section{NOMENCLATURE}

$P_{i n} \quad$ Input power (launched power) W

$\begin{array}{lll}P_{\text {rec }} \quad \text { Received power } & W\end{array}$

$P_{N L} \quad$ Overall nonlinearity lost Power $\quad W$

$P_{O N} \quad$ Power when channel is ON state $\quad W$

$\mu_{D 0 i} \quad$ Average power depletion $\quad W$

$D_{P 0} \quad$ Fractional power loss $\quad W$

$\sigma_{D 0 i} \quad$ Standard deviation of power loss $\quad \mathrm{dB}$

$W_{C} \quad$ Number of wavelength channels -

$N \quad$ Number of nodes $\quad$ -

$N_{A} \quad$ Number of amplifiers -

$G \quad$ Gain of the amplifier $\quad \mathrm{dB}$

$G_{R} \quad$ Raman gain coefficient $\mathrm{dB}$

$\Delta \beta \quad$ Phase-matching coefficient - 


\begin{tabular}{|c|c|c|}
\hline$T_{R}$ & Raman frequency shift & $\mathrm{THz}$ \\
\hline$\gamma_{P}$ & Peak Raman gain & $c m W^{-2}$ \\
\hline$\gamma_{i}$ & Coupling Raman gain coefficient for channels $1, i$ & - \\
\hline$L$ & Optical fiber length & $\mathrm{m}$ \\
\hline$P_{i n}$ & Input power & $\mathrm{dBm}$ \\
\hline$R_{b}$ & Bit rate & Gbps \\
\hline$B_{0}$ & Optical bandwidth & $\mathrm{GHz}$ \\
\hline$B_{e}$ & Electrical bandwidth & $\mathrm{GHz}$ \\
\hline$v$ & Operating frequency & $\mathrm{Hz}$ \\
\hline$f$ & Any frequency of any channel & $\mathrm{Hz}$ \\
\hline$\Delta \omega$ & Frequency channel spacing & $\mathrm{GHz}$ \\
\hline$\Delta \lambda$ & Wavelength spacing & $\mathrm{nm}$ \\
\hline$\lambda$ & Basic operating light wavelength & $\mathrm{nm}$ \\
\hline$n_{2}$ & Nonlinear refractive index & $m^{2} W^{-1}$ \\
\hline$n_{g}$ & Group index & $m^{2} W^{-1}$ \\
\hline$R_{d}$ & Responsivity of the receiver & $A W^{-1}$ \\
\hline$R_{L}$ & Load resistance & Ohm \\
\hline$F_{e}$ & Inversion factor & - \\
\hline$U$ & Degeneracy factor & - \\
\hline$T$ & Temperature & ${ }^{o} K$ \\
\hline$d$ & Walk-off parameter & - \\
\hline$L_{W}$ & Walk-off length & $\mathrm{m}$ \\
\hline$c$ & Speed of light in free space & $m s^{-1}$ \\
\hline$A_{\text {eff }}$ & Cross-sectional area of fiber core (effective) & $m^{2}$ \\
\hline$L_{e f f}$ & Effective Optical fiber length & $\mathrm{m}$ \\
\hline$B E R$ & Bit error rate & $\mathrm{dB}$ \\
\hline$X P M$ & Cross-phase modulation & - \\
\hline$F W M$ & Four-wave mixing & - \\
\hline$S R S$ & Stimulated Raman scattering & - \\
\hline
\end{tabular}


$I_{D} \quad$ Decision level of current for logic circuitry $\quad$ A

$\sigma_{1} \quad$ Standard deviation of lost power for bit

$\sigma \quad$ Standard deviation of lost power

Greek letters

$\alpha \quad$ Fiber loss coefficient (attenuation)

$\gamma \quad$ Nonlinearity coefficient of the fiber

$\Psi \quad$ To account for unstudied nonlinear effects

$\omega \quad$ Any angular frequency

$\beta \quad$ Dispersion of any order

$\sigma \quad$ Standard deviation of lost power

Subscript

$i \quad$ Channel number

$j \quad$ Channel number

$k \quad$ Channel number

$n \quad$ Index

$m \quad$ Index

accn Accumulated

ASE Amplified spontaneous emission

$o \quad$ Optical

$e \quad$ Electrical

c Chromatic

eff Effective

rboN Probability of both channels is ON

NL Nonlinear

D Decision level

${ }_{W} \quad$ Walk-off

Superscript

ch Any channel 